\title{
Policy-ready science
}

\author{
Although Nature Genetics generally urges authors to keep their claims within the research arena, basic research \\ occasionally turns up results that are ready for immediate application. In these cases we aim to assign some peer \\ referees familiar with the needs of policy makers and to provide accompanying commentary that puts the research into \\ an appropriate societal perspective.
}

$R$ esearch can stop the rot. In 1970 the Southern corn leaf blight was tackled by Arthur Lee Hooker et al. in their report "Reaction of corn seedlings with male-sterile cytoplasm to Helminthosporium maydis" (Plant Dis. Report. 54, 708-712, 1970). It turned out that plant breeders had prepared the field for the parasite, and the Texas cytoplasmic male-sterile (cms-T) mitochondrial genotype used to create most commercial F1 maize in the US was exquisitely susceptible to the fungus (better known as Cochliobolus heterostrophus (Drechsler)). Recognizing this fatal combination early was the key to arresting a costly error of monoculture. As in this example, research that isolates a necessary cause usually produces immediate attention and a change of policy.

A model of the difficulty with policy-ready research came from a model organism, when Nicolette Salmon Hillbertz et al. (Nat. Genet. 39, 1318-1320, 2007) found that the distinctive hair ridge in Ridgeback dogs results from the dominant effect of a duplication that was also a risk factor for a congenital defect: " 13 of 15 Ridgebacks with dermoid sinus were homozygous for the duplication." They made a policy recommendation: "The problem with dermoid sinus could be virtually eliminated by allowing ridgeless dogs in breeding and by avoiding matings between ridged dogs." The resulting arguments between journalists and dog breeders (http://www.canipur.nl/ user/image/ridge.pdf) illustrate how useful new information can get hyped, denied, minimized and finally incorporated into standard practice on new terms. More research in this area is unlikely to be forthcoming, but breeders may be motivated to sponsor studies to test the hypothesis (supported by several pedigrees) that with certain genetic or environmental modifiers the $R / R$ genotype can produce the breed-specific ridge without the defect (http:// camelotrr.com/2nd_ridge_article.pdf).

A human example we encountered this year found genetic markers of Native American ancestry to be a predictor of poor treatment outcome in childhood acute lymphoblastic leukemia (Jun Yang et al., Nat. Genet. 43, 237$241,2011)$. If this result can be independently replicated, it may become the basis for near-term clinical practice. As the accompanying News and Views commented (Chanock, Nat. Genet. 43, 178-179, 2011), the research result would become progressively more useful after refinement of proportional ancestry to specific genetic loci and then to causal pharmacogenetic mechanisms. Of course, more proof is better; the ideal "policy-ready" paper would contain internal independent replication via consortium research or parallel submission. However, these are hard to coordinate, and peer reviewing times in such situations begin to get unfeasibly long. In the meantime, we can instead get the basic research results into the public domain, with appropriate caveats, so that the results can be scrutinized, replicated and used.

In some professional fields, formal protocols have been established for the transfer of research results to improve practice. The most familiar of these is the use of randomized controlled clinical trials as the basis for updating medical guidelines and training. Change occurs most readily where there is a clear result that serves an unmet need, where a biomarker can be replaced by a test for a necessary causal agent, where the experimental design is statistically robust and protected from bias. A splendid example of such a trial is the work of Sankaranarayanan et al. (N. Engl. J. Med. 360, 1385-1394, 2009), who found that a single test for human papillomavirus administered to women aged 30-59 in a resource-poor environment in rural India led to a halving (hazard ratio 0.52 ) of the death rate from cervical cancer. The study not only suggests future epidemiological research but is also policy-ready.

In contrast, the recent reclassification by the International Agency for Research on Cancer (IARC) of radiofrequency electromagnetic fields from cell phones as "possibly carcinogenic to humans" (Lancet Oncol. 12, 624-625, 2011) on the basis of the largely negative evidence from the INTERPHONE study (Int. J. Epidemiol. 39, 675-694, 2010) has raised a lot of discussion in the news media about what the research is trying to do and on what timescale. What kind of answer do we need, and what measures should we take apart from using hands-free connections while we wait for definitive evidence? There is no doubt the available evidence was used transparently and responsibly by the expert panel at the IARC, but they tackled an intrinsically hard question and ran up against the limitations of the case-control experimental design and the evidence so far available.

One requirement of basic research is to isolate the experimental system in order to control its variables and gain clear results. Connecting the results back to the real world brings back in all the combinatorial difficulties of applied science, as well as the cultural differences of other stakeholders. From the perspective of decision makers (legislators and regulators), economic calculations are inevitable, but what many researchers do not realize is that decision makers often do not see any of the options open to them among the recommendations of the research paper. These options are legislation, publicity campaigns, economic incentives, warnings and disclaimers, mandatory monitoring and reporting, and changes to guidelines and practice.

While appreciating these limitations, we can make sure we provide the best scientific advice. For changing medical practice, translational research means shortening the path to the first human clinical trials. However, genetic research throws up many unpredictable susceptibilities, some of which can be immediately useful, and it is with these that we need to be most careful and alert. As with the cms-T maize and corn blight, genetic results almost always identify a combination of genetic and environmental factors resulting in a differential outcome for a specialized subset of the population-even for the most routine of situations; just consider the ubiquitous warning on soft drink containers, "Phenylketonurics: contains phenylalanine." 